\title{
Prediction of Cochlear Implant Outcomes in Patients With Prelingual Deafness
}

\author{
Dong Hoon Kang ${ }^{1}$ Myoung Jin Lee $\cdot$ Kyu-Yup Lee ${ }^{1} \cdot$ Sang Heun Lee ${ }^{2} \cdot$ Jeong Hun Jang ${ }^{1,3}$ \\ ${ }^{I}$ Department of Otorhinolaryngology, Kyungpook National University School of Medicine, Daegu; ${ }^{2}$ Department of Otorhinolaryngology, Daegu \\ Veterans Hospital, Daegu; ${ }^{3}$ Department of Otorhinolaryngology, Ajou University School of Medicine, Suwon, Korea
}

Objectives. To evaluate the factors that limit post-cochlear implantation (CI) speech perception in prelingually deaf children.

Methods. Patients with CI were divided into two groups according to Category of Auditory Performance (CAP) scores 3 years post-CI: the poor performance group (poor performance group, CAP scores $\leq 4, n=41$ ) and the good performance group (good performance group, CAP scores $\geq 5, n=85$ ). The distribution and contribution of the potential limiting factors related to post-CI speech perception was compared.

Results. Perinatal problems, inner ear anomalies, narrow bony cochlear nerve canal (BCNC), and intraoperative problems was significantly higher in the poor performance group than the good performance group $(P=0.010, P=0.003$, $P=0.001$, and $P=0.045$, respectively). The mean number of limiting factors was significantly higher in the poor performance group (1.98 \pm 1.04$)$ than the good performance group $(1.25 \pm 1.11, P=0.001)$. The odds ratios for perinatal problems and narrow bony cochlear nerve canal in the poor performance group in comparison with the good performance group were 4.878 ( $95 \%$ confidence interval, 0.067 to $0.625 ; P=0.005)$ and 4.785 ( $95 \%$ confidence interval, 0.045 to $0.972 ; P=0.046)$.

Conclusion. This study highlights the comprehensive prediction of speech perception after CI and provides otologic surgeons with useful information for individualized preoperative counseling of $\mathrm{CI}$ candidates.

Keywords. Cochlear Implantation; Sensorineural Hearing Loss; Speech Perception; Prognosis; Language Development

\section{INTRODUCTION}

Since cochlear implantation $(\mathrm{CI})$ became the standard procedure for managing severe to profound sensorineural hearing loss (SNHL), it has significantly enhanced speech perception in children with SNHL. Although post-CI improvement in speech perception is rapid in some patients, it is slow in others. Therefore, an investigation of patients exhibiting poor speech perception after $\mathrm{CI}$ is important, and the development of approaches for

- Received October 2, 2015

Revised November 3, 2015

Accepted November 9, 2015

- Corresponding author: Jeong Hun Jang

Department of Otorhinolaryngology, Ajou University School of Medicine,

164 World cup-ro, Yeongtong-gu, Suwon 16499, Korea

Tel: +82-31-219-5265, Fax: +82-31-219-5264

E-mail: jhj@ajou.ac.kr doing so has become a principal focus in this field.

Linguistic competence for complete speech perception is absent in most children with SNHL before and 1 year after CI. As experience in listening using the cochlear implant increases, speech perception generally improves. Pediatric cochlear implant users in particular exhibit enhanced auditory performance for up to 10 years after CI [1]. Because improved speech perception over time correlates with improved speech production and language, appropriate evaluation of speech perception in children after $\mathrm{CI}$ is important for optimal long-term post-CI performance.

Speech perception in children after CI depends on several factors including demographic and hearing characteristics and the features of the implant device [2]. Identification of the factors contributing to poor speech perception after $\mathrm{CI}$ is necessary, and several studies have addressed this issue [3,4]. Pre-CI as-

Copyright (C) 2016 by Korean Society of Otorhinolaryngology-Head and Neck Surgery.

This is an open-access article distributed under the terms of the Creative Commons Attribution Non-Commercial License (http://creativecommons.org/licenses/by-nc/4.0)

which permits unrestricted non-commercial use, distribution, and reproduction in any medium, provided the original work is properly cited. 
sessment of the responsible factors in each candidate will allow us to better predict outcomes after CI.

Retrospective multicenter studies have attempted to identify prognostic factors using a three-stage model of auditory performance that assesses speech perception over time [5]. However, these models were constructed for postlingually deaf adults who underwent $\mathrm{CI}$; consequently, age at the time of CI did not significantly affect post-CI outcomes. In prelingually deaf children, CI at earlier ages resulted in better speech perception than did CI at later ages [6]. In a study of children who underwent CI, preand post-implantation concerns were more evident in the poor speech perception group than the randomized good performance group; however, this study was limited by small sample size [7].

The aim of the present study was to analyze post-CI speech perception in prelingually deaf children according to factors known to limit speech perception. Two relatively large groups were compared to provide valuable information for preoperative counseling of CI candidates.

\section{MATERIALS AND METHODS}

This study was approved by the Institutional Review Board of the Clinical Research Institute of the Kyungpook National University Hospital (No. 2013-04-009-001). The requirement for informed consent was waived.

\section{Participants}

Between February 1999 and June 2009, of 242 patients who underwent CI in the Department of Otorhinolaryngology-Head and Neck Surgery at Kyungpook National University Hospital, 126 children were enrolled in the study. Patients who were followed up for less than 3 years or whose medical records were incomplete were excluded. Patients were divided into two groups according to their Category of Auditory Performance (CAP) scores 3 years post-CI. CAP is a nonlinear hierarchical scale of auditory receptive ability [8]. In this study, good performance was defined as the capability of understanding speech. Patients with CAP scores $\leq 4$ (can discriminate at least two speech sounds) comprised the poor performance group (poor performance group, $n=41$ ), and age- and sex-matched patients

\section{H I G G H L I I G}

- This study evaluated the effects of limiting gactors on speech perception after cochlear implantation (CI).

- The distribution of limiting factor is different from the contribution to post-CI outcome.

- The post-CI outcome should be comprehensively predicted based on the limiting factors. with CAP scores $\geq 5$ (can understand common phrases without lip reading) comprised the good performance group (good performance group, $n=85$ ). The mean follow-up times for the experimental and good performance groups were 99.6 and 105.6 months, respectively. Medical records including physical examinations, hearing status, temporal bone computed tomography, and surgery were reviewed retrospectively.

\section{Factors limiting for prognosis}

The literature was reviewed to identify well-established factors affecting CI prognosis; these are described in Table 1.

Delayed CI in childhood was defined as CI performed in children between 5-years-old and 10-years-old. Perinatal problems included hyperbilirubinemia, meningitis, low birth weight, and other etiologies previously associated with SNHL. Inner ear anomalies were evaluated according to the classification system proposed by Sennaroglu [9]. Use of a hearing aid for over 3 months was considered a prerequisite for $\mathrm{CI}$, as per the recommendation of the National Health Insurance Service. Limiting factors also included comorbid disorders related to educational level, which has been linked to poor speech performance after CI, and long-term deafness. Although long-term deafness can partially overlap delayed CI, it has been shown to reduce postCI speech perception.

Using the bony cochlear nerve canal (BCNC) evaluation system developed by Fatterpekar et al. [10], relationships between BCNC parameters and both hearing level and the presence of the cochlear nerve were established [11]. In our study, the width of the BCNC at the fundus of the internal auditory canal was determined in axial slices of temporal bone computed tomography images, at the point where it was maximal in contiguous sections, by measuring the distance between the inner margins of the bony wall at the mid-portion. BCNC width was a prognostic parameter in this study. A narrow BCNC was defined as a width $\leq 1.40 \mathrm{~mm}$ [12]. Intraoperative events with the potential to affect $\mathrm{CI}$ outcomes were considered to be limiting factors.

The mean thresholds of preoperative pure tone audiograms were $101.1 \pm 10.1 \mathrm{~dB}$ HL in the poor performance group and $98.3 \pm 6.7 \mathrm{~dB} H \mathrm{HL}$ in the good performance group. Although this difference showed a trend toward significance $(P=0.069)$, the absolute value of each group was not appropriate for residual hearing, and preoperative residual hearing level was therefore not included as a limiting factor.

\section{Data analysis}

The distribution of the limiting factors was compared between the two groups using the chi-square test and Student $t$-test. Logistic regression analysis was performed and odds ratios were calculated to assess the contribution of the various limiting factors to post-CI outcome. All analyses were performed using SPSS ver. 17.0 (SPSS Inc., Chicago, IL, USA). The $P$-values $<0.05$ indicated statistical significance. 
Table 1. Limiting factors affecting $\mathrm{Cl}$ outcome

\begin{tabular}{ll}
\hline Factor & \multicolumn{1}{c}{ Description } \\
\hline Delayed $\mathrm{Cl}$ in childhood & Cl between 5 and 10 years old \\
Perinatal problems & Low birth weight, meningitis, hyperbilirubinemia, etc. \\
Inner ear anomalies & Criteria defined by Sennaroglu [9] \\
Hearing aid nonuse or short term use & Duration of use less than 3 months \\
Comorbid disorders & Autism, mental restriction, etc. \\
Long-term deafness & Duration of deafness $\geq 10$ years before $\mathrm{Cl}$ \\
Bony cochlear nerve canal & Measurement defined by Fatterpekar et al. [10] \\
& Narrow bony cochlear nerve canal in case of the width $\leq 1.40 \mathrm{~mm}$ \\
Intraoperative problems & Perilymph gusher, incomplete electrode insertion, cochlear ossification, absence of neural response \\
\hline
\end{tabular}

$\mathrm{Cl}$, cochlear implantation.

Table 2. Demographics for the poor performance group and the good performance group

\begin{tabular}{lcc}
\hline Variable & $\begin{array}{c}\text { Poor performance } \\
\text { group }(n=41)\end{array}$ & $\begin{array}{c}\text { Good performance } \\
\text { group }(n=85)\end{array}$ \\
\hline Male:female & $26: 15$ & $50: 35$ \\
Right:left & $21: 20$ & $47: 38$ \\
Device & Nucleus & Nucleus \\
Mean age at the time of Cl (yr) & $11.5 \pm 1.8$ & $11.5 \pm 1.3$ \\
CAP score & $0(n=1)$ & $5(n=25)$ \\
& $2(n=4)$ & $6(n=45)$ \\
& $4(n=36)$ & $7(n=15)$ \\
\hline
\end{tabular}

$\mathrm{Cl}$, cochlear implantation; CAP, categories of auditory performance.

\section{RESULTS}

The demographics of the experimental and good performance groups are summarized in Table 2. The mean CAP score was $3.70 \pm 0.84$ in the former and $5.88 \pm 0.68$ in the latter. The most common etiology was congenital SNHL in both groups, followed by meningitis and inner ear anomalies.

The distribution of limiting factors was compared between the two groups (Table 3). The number of perinatal problems, inner ear anomalies, narrow BCNC, and intraoperative complications was significantly higher in the poor performance group than the good performance group $(P=0.010, P=0.003, P=0.001$, and $P=0.045$, respectively). The mean number of limiting factors differed significantly $(P=0.001)$ between the experimental $(1.98 \pm$ $1.04)$ and control (1.25 \pm 1.11$)$ groups.

\section{Cochlear implantation timing}

The experimental and good performance groups had a similar incidence of delayed CI ( $n=15,36.6 \%$ and $n=34,40.0 \%$, respectively). The mean duration of deafness in cases of delayed CI was $80.1 \pm 21.8$ months in the poor performance group $(n=15)$ and $82.8 \pm 18.0$ months in the good performance group $(n=34)$. The mean age at the time of $C I$ was $7.7 \pm 1.9$ years in the poor performance group and $7.2 \pm 1.3$ years in the good performance group.

In the 19 patients with long-term deafness, the mean duration
Table 3. Comparison of limiting factors between 2 groups

\begin{tabular}{lccc}
\hline Factor & $\begin{array}{c}\text { Poor } \\
\text { performance } \\
\text { group }(\mathrm{n}=41)\end{array}$ & $\begin{array}{c}\text { Good } \\
\text { performance } \\
\text { group }(\mathrm{n}=85)\end{array}$ & P-value \\
\hline $\begin{array}{l}\text { Delayed cochlear } \\
\text { implantation in childhood }\end{array}$ & $15(36.6)$ & $34(40.0)$ & 0.713 \\
$\begin{array}{l}\text { Perinatal problems } \\
\text { Inner ear anomalies }\end{array}$ & $18(43.9)$ & $17(20.0)$ & 0.010 \\
$\begin{array}{l}\text { Hearing aid nonuse or } \\
\text { short-term use }\end{array}$ & $14(34.1)$ & $9(10.6)$ & 0.003 \\
$\begin{array}{l}\text { Comorbid disorders } \\
\text { Long-term deafness }\end{array}$ & $5(12.2)$ & $3(2.4)$ & 0.976 \\
Narrow bony cochlear nerve & $11(26.8)$ & $3(3.5)$ & 0.112 \\
$\quad$ canal & $14(34.1)$ & $15(17.6)$ & 0.045 \\
\hline Intraoperative problems & $14(16.5)$ & 0.605 \\
\hline
\end{tabular}

Values are presented as number (\%).

of deafness was $138.4 \pm 21.3$ months in the poor performance group ( $\mathrm{n}=5,12.2 \%)$ and $136.9 \pm 15.2$ months in the good performance group $(n=14,16.5 \% ; P>0.05)$. The mean age at the time of $\mathrm{CI}$ was $11.5 \pm 1.8$ years in the poor performance group and $11.5 \pm 1.3$ years in the good performance group $(P>0.05)$.

\section{Perinatal problems and comorbid disorders}

Eighteen patients $(43.9 \%)$ in the poor performance group had perinatal problems or comorbid disorders compared with 17 $(20 \%)$ in the good performance group. Febrile illness, including meningitis, was the most frequent disorder in both groups. Six patients $(12.5 \%)$ in the poor performance group had comorbid disorders compared with three $(3.4 \%)$ in the good performance group. Comorbid disorders in the poor performance group included Waardenburg syndrome, autism, mitochondrial myopathy, renal disease, mental restriction, developmental delay, and epilepsy. In the good performance group, two patients exhibited developmental delay and one patient had an arachnoid cyst.

\section{Inner ear anomalies}

Inner ear anomalies were more frequent in the poor performance group than the good performance group $(P=0.003)$. In the poor performance group, inner ear anomalies included in- 
Table 4. Estimation of limiting factors

\begin{tabular}{lcc}
\hline Factor & P-value & Exp (B) (95\% confidence interval) \\
\hline Delayed cochlear implantation in childhood & 0.260 & $1.875(0.628-5.598)$ \\
Perinatal problems & 0.005 & $0.205(0.067-0.625)$ \\
Inner ear anomalies & 0.120 & $0.356(0.097-1.311)$ \\
Poor hearing aid compliance & 0.731 & $1.703(0.082-35.426)$ \\
Comorbid disorders & 0.065 & $0.165(0.025-1.116)$ \\
Long-term deafness & 0.561 & $1.550(0.354-6.777)$ \\
Narrow bony cochlear nerve canal & 0.046 & $0.209(0.045-0.972)$ \\
Intraoperative problems & 0.114 & - \\
\hline
\end{tabular}

complete partition (IP) (IP-I, $\mathrm{n}=2$; IP-II, $\mathrm{n}=6$; and IP-III, $\mathrm{n}=2$ ), cochlear hypoplasia $(n=3)$, common cavity $(n=1)$, and enlarged vestibular aqueducts $(n=7)$. Ear anomalies in the good performance group included IP-II $(n=4)$, common cavity $(n=2)$, and enlarged vestibular aqueducts $(n=12)$.

\section{$\mathrm{BCNC}$}

The mean BCNC width on the side of the cochlear implant was significantly smaller in the poor performance group $(1.67 \pm 0.99$ $\mathrm{mm})$ than the good performance group $(2.16 \pm 0.58 \mathrm{~mm}$, $P=0.010$ ), as was the mean width on the contralateral side $(1.65 \pm 0.99$ and $2.19 \pm 0.59 \mathrm{~mm}$, respectively; $P=0.005)$. Both the proportions of patients with a narrow BCNC was more frequent in the poor performance group $(n=11)$ than the good performance group $(\mathrm{n}=3, P=0.001)$.

\section{Intraoperative problems}

Intraoperative problems included perilymph gusher, incomplete electrode insertion, cochlear ossification, and absence of a neural response (14 patients [34.1\%] in the poor performance group and $15[17.6 \%]$ in the good performance group, $P=$ 0.045 ). The numbers of perilymph gushers, incomplete electrode insertions, and cochlear ossifications in the poor performance group were 8,3 , and 3 , respectively; in the good performance group, they were 12,2 , and 1 , respectively.

\section{Relative risk of the limiting factors}

In a multiple logistic regression, the odds ratios for perinatal problems and narrow BCNC in the poor performance group compared with the good performance group were 4.878 (95\% confidence interval, 0.067 to $0.625 ; P=0.005), 4.785$ (95\% confidence interval, 0.045 to $0.972 ; P=0.046$ ), respectively (Table 4).

\section{DISCUSSION}

In this study, the limiting factors affecting post-CI speech perception were compared in poor performers and good performers via a retrospective chart review.

The existence of a critical period for language development in children has been well documented; their best opportunity to learn language is during their first 5 years of life. The critical period for $\mathrm{CI}$, in terms of language development, in children with profound SNHL is $<3.5-5$ years of age. Considering that even in adults with prelingual deafness significantly improved speech perception scores after $\mathrm{CI}$ compared with preimplantation scores in the study by Teoh et al. [13], as much more did CI during the critical period. Therefore, the time at which $\mathrm{CI}$ is performed is a potential limiting factor for post-CI speech performance in prelingually deaf patients.

Speech understanding in patients with long-term prelingual deafness significantly improved after $\mathrm{CI}$ in several studies, although performance levels varied widely. Nonetheless, longterm deafness negatively affects post-CI speech perception. Arisi et al. [14] reported that CI significantly delayed deafness in poor performing adolescents with prelingual deafness. In our study, long-term deafness was similarly distributed between both groups, and thus was not a significant limiting factor. However, the mean age at diagnosis of deafness was $41.8 \pm 10.0$ years in the 5 long-term deaf patients in the poor performance group and $28.7 \pm 12.4$ years in the 14 long-term deaf patients in the good performance group $(P=0.052)$. Moreover, the mean number of limiting factors was larger in the 19 patients with longterm deafness $(2.7 \pm 0.9)$ compared with the other 107 patients $(1.3 \pm 1.0)$ and in the long-term deaf patients in the poor performance group (3.4 \pm 0.9$)$ compared with those in the good performance group $(2.5 \pm 0.88, P=0.053)$. The differences in the frequency of inner ear anomalies $(P=0.084)$ and narrow BCNC $(P=0.065)$ between patients with and without long-term deafness showed a trend toward significance. These differences might reflect the difference in the CAP scores in patients with and without long-term deafness $(4.0 \pm 0.0$ and $5.4 \pm 0.5$, respectively, $P<0.001)$.

Age at the time of $\mathrm{CI}$ affects speech perception in children after $\mathrm{CI}$, and younger children displayed more rapid post-CI improvement than older children in one study [15]. In our study, the percentage of patients with delayed CI was similar in the experimental $(n=15,36.6 \%)$ and control $(n=34,40.0 \%)$ groups, in agreement with previous studies showing no effect of delayed $\mathrm{CI}$ on post-CI speech perception [15]. The mean age at diagnosis of deafness in patients with delayed CI was $33.1 \pm 14.9$ months in the poor performance group and $27.2 \pm 15.3$ months in the 
good performance group $(P=0.220)$. Perinatal problems $(P=0.069)$ was more frequent in the poor performance group than the good performance group, and the mean thresholds of preoperative pure tone audiograms were 106.4 $\pm 13.6 \mathrm{~dB} \mathrm{HL}$ and 98.2 $\pm 9.2 \mathrm{~dB} \mathrm{HL}$, respectively $(P=0.047)$. These differences might account for the post-CI speech perception differences in patients with delayed CI of both groups.

Perinatal problems differed significantly between the experimental and good performance groups. The most common problems were infections such as meningitis and hyperbilirubinemia and prematurity (low birth weight). Philippon et al. [16] recommended early $\mathrm{CI}$ for patients with bilateral profound deafness secondary to meningitis owing to the risk of labyrinthitis ossificans. Blamey et al. [17] reported below average post-CI speech perception in postlingually deaf adults with meningitis, perhaps due to reduced survival of ganglion cells. In our study, infection was one of the most frequent problems, thus suggesting that perinatal disorders are significant limiting factors. Hyperbilirubinemia and prematurity are classic risk factors for auditory neuropathy spectrum disorder, and post-CI speech perception in patients with hyperbilirubinemia or low birth weight is variable but tends toward favorable.

Numerous inner ear anomalies cause unwanted postsurgical outcomes. They are associated with the cochleostomy site, inadequate electrode placement and stability, facial nerve injury, perilymph leakage, and post-CI meningitis. It is universally accepted that children with more severe inner ear anomalies have poorer hearing outcomes after $\mathrm{CI}$ than those with less severe anomalies. Papsin [18] reported that speech perception did not differ significantly in children with anomalous and normal cochleae and that children with narrowing of the internal auditory/cochlear canals, common cavities, and cochlear hypoplasia had worse post-CI speech perception than those with other anomalies. In our study, the frequency of inner ear anomalies, cochlear aplasia, and common cavities was higher in the poor performance group than the good performance group, in agreement with Papsin [18]; however, these anomalies did not significantly influence post-CI speech perception.

At present, children with additional disabilities routinely undergo $\mathrm{CI}$, with the aim of enhancing their interactions with the environment by improving their understanding of language and speech development. Pyman et al. [19] found that post-CI speech perception was lower in children with motor and/or cognitive delays compared with other children and emphasized that central factors account in large part for variations in the outcomes of pediatric CI. Conversely, Wiley et al. [20] reported that $\mathrm{CI}$ improved the communication skills of children with multiple handicaps and that their families would opt for $\mathrm{CI}$ again if need be. Although comorbid disorders can negatively affect speech perception after $\mathrm{CI}$, auditory rehabilitation of children with comorbid disorders should be performed on an individual basis, and the method used to assess the postoperative development of speech perception should be selected according to the type of comorbid disorder present.

A perilymph gusher is the pulsatile egress of clear fluid for up to 1 minute during cochleostomy. It differs from perilymph leakage, which commonly occurs after cochleostomy associated with $\mathrm{CI}$, and is generally nonpulsatile. The likelihood of a perilymph gusher increases when cochleovestibular anomalies are present. However, Adunka et al. [21] suggest that the type of labyrinthine anomaly has a greater effect on postoperative performance than the presence or absence of an intraoperative perilymph gusher. Therefore, rather than concluding that a perilymph gusher has a significant impact on speech perception after CI, the correlation between it and auditory perception in patients with inner ear anomalies should be explored. Incomplete electrode insertion may closely correlate with poor post-CI speech perception. In our study, patients in the poor performance group with incomplete electrode insertion exhibited cochlear hypoplasia, postmeningitic ossification, and vestibular insertion. Postmeningitic ossification was evident in only one patient in the poor performance group and two patients in the good performance group. Reducing the number of intracochlear sites available for stimulation limits the size of the stimulated neural population. Lazard et al. [5] reported that electrode activity $>85 \%$ significantly improved speech perception. In our study, intraoperative problems were more frequent in the poor performance group than the good performance group but did not limit speech perception after $\mathrm{CI}$, which suggests the further evaluation of surgical proficiency as a prognostic factor.

Estimation of cochlear nerve integrity may be of fundamental importance when selecting candidates for surgery. The frequency of narrow BCNCs was significantly different and was a significant limiting factor between the two groups. Therefore, BCNC width might substantially effect speech perception after CI, although studies of the relationship between BCNC and post-CI outcome are rare [22].

Most patients in this study wore the hearing aid immediately after SNHL was diagnosed, which might indirectly support the importance of early diagnosis of SNHL.

Since postoperative factors such as the number of active electrodes, insertion depth, and MAP data were not unavailable for all patients, we focused on the preoperative and intraoperative factors related to speech perception after CI to increase the reliability of the data. The findings in our study may provide otologic surgeons with useful information for individualized preoperative counseling of $\mathrm{CI}$ candidates and their families.

In summary, this study evaluated the effects of several limiting factors on speech perception after CI. The different distributions of the limiting factors between the experimental and good performance groups should not be interpreted as differences in their contribution to post-CI speech perception. This study highlights the complexity and difficulties associated with the prediction of speech perception after CI based on limiting factors. 


\section{CONFLICT OF INTEREST}

No potential conflict of interest relevant to this article was reported.

\section{ACKNOWLEDGMENTS}

This work was supported by Biomedical Research Institute grant, Kyungpook National University Hospital, Daegu, Republic of Korea in 2015.

\section{REFERENCES}

1. Beadle EA, McKinley DJ, Nikolopoulos TP, Brough J, O’Donoghue GM, Archbold SM. Long-term functional outcomes and academicoccupational status in implanted children after 10 to 14 years of cochlear implant use. Otol Neurotol. 2005 Nov;26(6):1152-60.

2. O'Donoghue GM, Nikolopoulos TP, Archbold SM. Determinants of speech perception in children after cochlear implantation. Lancet. 2000 Aug 5;356(9228):466-8.

3. Gomaa NA, Rubinstein JT, Lowder MW,Tyler RS, Gantz BJ. Residual speech perception and cochlear implant performance in postlingually deafened adults. Ear Hear. 2003 Dec;24(6):539-44.

4. Green KM, Bhatt Y, Mawman DJ, O’Driscoll MP, Saeed SR, Ramsden RT, et al. Predictors of audiological outcome following cochlear implantation in adults. Cochlear Implants Int. 2007 Mar;8(1):1-11.

5. Lazard DS, Vincent C, Venail F, Van de Heyning P, Truy E, Sterkers O, et al. Pre-, per- and postoperative factors affecting performance of postlinguistically deaf adults using cochlear implants: a new conceptual model over time. PLoS One. 2012;7(11):e48739.

6. Svirsky MA, Teoh SW, Neuburger H. Development of language and speech perception in congenitally, profoundly deaf children as a function of age at cochlear implantation. Audiol Neurootol. 2004 Jul-Aug;9(4):224-33.

7. Gordon KA, Daya H, Harrison RV, Papsin BC. Factors contributing to limited open-set speech perception in children who use a cochlear implant. Int J Pediatr Otorhinolaryngol. 2000 Dec;56(2):101-11.

8. Archbold S, Lutman ME, Marshall DH. Categories of Auditory Performance. Ann Otol Rhinol Laryngol Suppl. 1995 Sep;166:312-4.

9. Sennaroglu L. Cochlear implantation in inner ear malformations: a review article. Cochlear Implants Int. 2010 Mar;11(1):4-41.

10. Fatterpekar GM, Mukherji SK, Alley J, Lin Y, Castillo M. Hypoplasia of the bony canal for the cochlear nerve in patients with congenital sensorineural hearing loss: initial observations. Radiology. 2000 Apr;215(1):243-6.

11. Jang JH, Kim JH, Yoo JC, Kim CH, Kim MS, Chang SO, et al. Implication of bony cochlear nerve canal on hearing in patients with congenital unilateral sensorineural hearing loss. Audiol Neurootol. 2012;17(5):282-9.

12. Adunka OF, Jewells V, Buchman CA.Value of computed tomography in the evaluation of children with cochlear nerve deficiency. Otol Neurotol. 2007 Aug;28(5):597-604.

13. Teoh SW, Pisoni DB, Miyamoto RT. Cochlear implantation in adults with prelingual deafness: part I. clinical results. Laryngoscope. 2004 Sep;114(9):1536-40.

14. Arisi E, Forti S, Pagani D, Todini L, Torretta S, Ambrosetti U, et al. Cochlear implantation in adolescents with prelinguistic deafness. Otolaryngol Head Neck Surg. 2010 Jun;142(6):804-8.

15. Zwolan TA, Ashbaugh CM, Alarfaj A, Kileny PR, Arts HA, El-Kashlan HK, et al. Pediatric cochlear implant patient performance as a function of age at implantation. Otol Neurotol. 2004 Mar;25(2):11220.

16. Philippon D, Bergeron F, Ferron P, Bussieres R. Cochlear implantation in postmeningitic deafness. Otol Neurotol. 2010 Jan;31(1):837.

17. Blamey P, Arndt P, Bergeron F, Bredberg G, Brimacombe J, Facer G, et al. Factors affecting auditory performance of postlinguistically deaf adults using cochlear implants. Audiol Neurootol. 1996 SepOct;1(5):293-306.

18. Papsin BC. Cochlear implantation in children with anomalous cochleovestibular anatomy. Laryngoscope. 2005 Jan;115(1 Pt 2 Suppl 106):1-26.

19. Pyman B, Blamey P, Lacy P, Clark G, Dowell R. The development of speech perception in children using cochlear implants: effects of etiologic factors and delayed milestones. Am J Otol. 2000 Jan;21(1): 57-61.

20. Wiley S, Jahnke M, Meinzen-Derr J, Choo D. Perceived qualitative benefits of cochlear implants in children with multi-handicaps. Int J Pediatr Otorhinolaryngol. 2005 Jun;69(6):791-8.

21. Adunka OF, Teagle HF, Zdanski CJ, Buchman CA. Influence of an intraoperative perilymph gusher on cochlear implant performance in children with labyrinthine malformations. Otol Neurotol. 2012 Dec;33(9):1489-96.

22. PagarkarW, Gunny R, Saunders DE, Yung W, Rajput K. The bony cochlear nerve canal in children with absent or hypoplastic cochlear nerves. Int J Pediatr Otorhinolaryngol. 2011 Jun;75(6):764-73. 